\section{Implantation of refractive multifocal intraocular lens with a surface-embedded near section for cataract eyes complicated with a coexisting ocular pathology}

M Ouchi ${ }^{1,2}$ and S Kinoshita ${ }^{2}$

\begin{abstract}
Purpose To evaluate the postoperative outcomes of cataract eyes complicated with coexisting ocular pathologies that underwent implantation of a refractive multifocal intraocular lens (MIOL) with a surfaceembedded near section.

Methods LENTIS MPlus (Oculentis GmbH) refractive MIOLs were implanted in 15 eyes with ocular pathologies other than cataract (ie, six high-myopia eyes with an axial length longer than $28 \mathrm{~mm}$, two fundus albipunctatus eyes, two branch retinal-vein occlusion eyes, four glaucoma eyes (one with high myopia), and two keratoconus eyes). Uncorrected or corrected distance and near visual acuity (VA) (UDVA, UNVA, CDVA, and CNVA), contrast sensitivity, and defocus curve were measured at 1 day and 6 months postoperatively, and each patient completed a 6-month postoperative questionnaire regarding vision quality and eyeglass use.

Results Thirteen eyes $(87 \%)$ registered 0 or better in CDVA and 12 eyes (73\%) registered better than 0 in CNVA. Contrast sensitivity in the eyes of all patients was comparable to that of normal healthy subjects. No patient required eyeglasses for distance vision, but three patients $(20 \%)$ required them for near vision. No patient reported poor or very poor vision quality. Conclusion With careful case selection, sectorial refractive MIOL implantation is effective for treating cataract eyes complicated with ocular pathologies.

Eye (2015) 29, 649-655; doi:10.1038/eye.2015.12; published online 6 March 2015
\end{abstract}

Introduction

At present, two types of multifocal intraocular lenses (MIOLs) are available, diffractive MIOLs and refractive MIOLs, and careful patient selection and choice of lens type are key for the successful use of MIOLs. ${ }^{1}$ In refractive MIOLs, halo or glare symptoms are more prominent than in diffractive MIOLs because of light scattering at the transitional zone between the distant and near focus of the MIOL. ${ }^{2}$ In addition, the near visual acuity (VA) when using refractive MIOLs tends to depend on pupil size because of the near focus zone of the MIOL being concentrically allocated. ${ }^{3}$ On the other hand, one underlying problem associated with diffractive MIOLs is the reduced contrast post implantation due to the optical feature of the lens. ${ }^{4-6}$

The LENTIS MPlus (Oculentis GmbH, Berlin, Germany) is an acrylic refractive MIOL that was designed on the basis of refractive rotational asymmetry. The aim of that design is to reduce sources of light scattering and aberrations that cause disturbing reflections, halos, and glare; several studies have reported the clinical results of that lens design. ${ }^{7-10}$ Although studies have reported that the postoperative contrast sensitivity outcomes using other MIOLs are worse than those with monofocal IOLs, ${ }^{11,12}$ Alió et $a l^{8}$ reported that the postoperative contrast sensitivity outcomes with MPlus are comparable to those of monofocal IOLs.

Although the use of diffractive MIOLs has become increasingly popular because of their
${ }^{1}$ Ouchi Eye Clinic, Kyoto, Japan

${ }^{2}$ Department of Ophthalmology, Kyoto Prefectural University of Medicine, Kyoto, Japan

Correspondence: M Ouchi, Ouchi Eye Clinic 47-1 Karahashi Rajomoncho, Minami-ku, Kyoto 601-8453, Japan

Tel: +81 756627117 Fax: +8175662 7118 E-mail: mouchi@skyblue. ocn.ne.jp

Received: 29 July 2014 Accepted in revised form: 24 December 2014 Published online: 6 March 2015 
good reading-distance VA and their option of variably adding power for near vision, some patients, even those with healthy eyes, complain of hazy vision, which might be the result of reduced contrast. Therefore, it is known that careful consideration must be taken when deciding whether to implant MIOLs in elderly patients over 80 years of age or in patients with corneal or retinal diseases. Ophthalmic surgeons debate on whether or not to implant MIOLs in interdisciplinary cases such as patients with early-stage glaucoma, high myopia with good corrected VA, or mild keratoconus, because, unlike with monofocal IOL implantation in such subclinical handicapped cases, MIOL implantation can result in a decrease in the patient's visual function because of the precision of the lens. However, the use of refractive MIOLs has the potential advantage of avoiding light scattering due to diffraction and less light energy loss, and thus it is feasible that those lenses can be implanted in such cases. Although there are reported clinical results of MPlus implantation in cases of anisometropic amblyopia, ${ }^{13}$ there has yet to be a report regarding MPlus implantation in eyes afflicted with organic eye disease.

The purpose of this study was to evaluate the clinical results of MPlus sectorial refractive MIOL implantation in eyes with cataract complicated with a coexisting ocular pathology.

\section{Patients and methods}

\section{Patients}

This prospective, consecutive, nonrandomized, interventional, clinical case study involved 15 eyes of 11 patients ranging in age from 23 to 72 years (mean age: $51.4 \pm 11.84$ years (mean $\pm S D)$ ), with cataract complicated with other ocular pathologies possibly affecting visual function, who underwent cataract surgery with implantation of a sectorial refractive MIOL. Eyes with an axial length longer than $28 \mathrm{~mm}$ were considered as highmyopia eyes, and eyes in which choroidal

neovascularization was detected by optical coherence tomography (OCT) were excluded from the study. Eyes showing apical decentration, asymmetric bowtie, and indices of keratoconus in corneal topography images were diagnosed as keratoconus. Eyes with both optic disc cupping of cupping/disc ratio $>0.6$ and glaucomatous visual field defect detected using a perimeter (Octopus 900; Haag-Streit AG, Koeniz, Switzerland) were included in the glaucoma eyes. Other fundus diseases were diagnosed by funduscopic examination, fluorescein angiography, and/or OCT depending on the disease. Prior informed consent was obtained from all patients, and this study was performed in accordance with the tenets set forth in the Declaration of Helsinki and was approved by the ethical committee of Ouchi Eye Clinic, Kyoto, Japan.

\section{MIOL and calculation}

The MIOLs used in this study were the LENTIS MPlus LS-313MF30 and the LENTIS MPlus Toric LU-313MFT (Oculentis). Both MIOLs are biconvex plate haptic acrylic MIOLs that have an aspheric distance-vision zone combined with sector-shaped near-vision zone of 3.0 diopters (D) addition. Theoretically, this design makes the IOL independent of pupil size for near VA. The toric model is also provided in the same platform. Available D choices range from 0 to $36.0 \mathrm{D}$ with $0.01 \mathrm{D}$ steps in spherical power, and from $0.25 \mathrm{D}$ to $12.0 \mathrm{D}$ with $0.01 \mathrm{D}$ steps in cylinder power. The IOL spherical power was calculated using the Haigis formula on an optical biometer (IOLMaster; Carl Zeiss Meditec AG, Jena, Germany). Data for the IOL calculations were taken from direct use of the optical biometry (IOLMaster; Carl Zeiss Meditec AG) or from ultrasonic immersion biometry data in cases with a dense cataract. The IOL cylinder power and alignment axis were calculated and provided by the product manufacturer taking into account the IOLMaster keratometry readings, as well as mandatory data input on the incision location and an estimate of the surgically induced astigmatism.

\section{Surgical technique}

For the cases requiring the toric model, three marks (two horizontal and one vertical) were made along the limbus prior to surgery using a 24-guage $(G)$ needle with marker ink (Gentian Violet Marker Pad; Becton, Dickinson and Co., Franklin Lakes, New Jersey, NY, USA) with the patient in a sitting position. At the beginning of surgery, the steepest meridian was marked with a toric-IOL corneal gauge and a toric-IOL axis marker (both from Duckworth and Kent Ltd., Baldock, United Kingdom). A 2.2-mm bent clear corneal incision was made using a 2.2-mm disposable steel knife (Slit knife 2.2DB; Alcon Laboratories, Inc., Fort Worth, TX, USA). Phacoemulsification was performed using the Infiniti Vision System (Alcon Laboratories) with an attached $0.9-\mathrm{mm}$ mini flare ABS phaco tip, $45^{\circ}$ KELMAN, and ultra-sleeve (Alcon Laboratories). After the capsule was filled with the viscoelastic agent, a LENTIS MPlus or LENTIS MPlus Toric IOL was inserted using a Viscoject-injector affixed with a Viscoglide 2.2 cartridge (both from Oculentis). In the toric model, the IOL was rotated to adjust its toric mark onto the corneal axis mark that was marked at the start of surgery under irrigation with a 22-G irrigating cannula (MST, Redmond, WA, USA) after removal of the viscoelastic material. Of the 15 eyes, the toric model IOLs were 
implanted in 9 eyes of 7 patients, and there were no intraoperative complications.

\section{Outcome measures}

Preoperatively, all patients underwent a full ophthalmic examination including refractive status, distance VA slitlamp evaluation, pupil size of photopic condition, tonometry, fundoscopy, and a visual field test; corneal topography and optical coherence tomography (OCT) were performed according to need in some cases. The schedule of postoperative examinations was as follows: uncorrected and corrected distance VA (UDVA and CDVA, respectively) and uncorrected and corrected near VA (UNVA and CNVA, respectively) were evaluated at 1 day and 6 months postoperatively, and other evaluations including contrast sensitivity testing, measurements of VA from intermediate to near, and defocus curve were performed at 6 months postoperatively. Refractive status and corneal astigmatism were examined using an autorefractor/ keratometer (ARK-560 A; Nidek Co., Ltd, Gamagori, Japan). Contrast sensitivity was evaluated with best distance correction without glare under a mesopic condition using a vision testing instrument (CSV-1000; VectorVision, Greenville, $\mathrm{OH}, \mathrm{USA})$. In addition to UDVA, BDVA, UNVA, and CNVA, near-to-intermediate distance VA was measured using a multi-distance chart (TMI-V5; Precision Vision, La Salle, IL, USA) that is set with VA sheets for $1 \mathrm{~m}$, and for 70,50, 40, and $30 \mathrm{~cm}$; uncorrected VA and distance-corrected VA were measured using these sheets. The defocus curve data were obtained at a distance of $5 \mathrm{~m}$ with monocular vision and the patient's distance correction. Spherical lenses were added in $0.5 \mathrm{D}$ steps from +2.0 to $-6.0 \mathrm{D}$, and VA was recorded for each type of blur. All recorded information was represented in a 2-dimensional graphic.

At the final follow-up visit (ie, 6 months postoperatively), all patients completed a questionnaire pertaining to the following: (1) spectacle use for distance and near vision (response scale: $1=$ never, $2=$ occasionally, and 3 =always) and (2) overall opinion of the quality of distance and near vision (response scale: $1=$ very poor, 2 = poor, 3 =acceptable, $4=$ good, and $5=$ very good).

\section{Results}

\section{Preoperative baseline data}

All enrolled patients completed all of the scheduled examinations. The mean preoperative CDVA and BDVA were $1.35 \pm 0.51(\log M A R)$ and $0.27 \pm 0.47(\log$ MAR), respectively. Mean preoperative refractive equivalent and corneal astigmatism were $-9.48 \pm 6.64 \mathrm{D}$ and $1.38 \pm 0.55 \mathrm{D}$, respectively. The toric model IOL was implanted into 13 of the 15 eyes. The mean axial length was $26.5 \pm 2.55 \mathrm{~mm}$ and the mean photopic pupil diameter was $3.82 \pm 0.61$ (2.70-4.62) $\mathrm{mm}$. The breakdown of the coexisting diseases is as follows: high myopia with axial length longer than $28 \mathrm{~mm}$ (six eyes of four patients), fundus albipunctatus (two eyes of one patient), branch retinal vein occlusion (two eyes of two patients), a past history of acute angleclosure glaucoma of Aulhorn classification stage 3 (one eye of one patient), normal-tension glaucoma of Aulhorn classification stage 1 (three eyes of two patients; one of those three eyes was also high myopia), and keratoconus (two eyes of two patients) (Table 1).

Table 1 Preoperative Patient data

\begin{tabular}{llllllll}
\hline Case & Age & Sex & CDVA & CC & SE & Pupil Size & Complication \\
\hline 1 & 47 & M & 0.9 & 0.75 & -20.125 & 3.84 & High Myopia \\
2 & 47 & M & 0.8 & 1.25 & -21.875 & 3.82 & High Myopia \\
3 & 41 & F & 0.5 & 2.5 & -0.125 & 4.62 & Keratoconus \\
4 & 49 & F & 0.6 & 1.5 & -4.375 & 2.7 & BRVO \\
5 & 55 & M & 1.5 & 1.5 & -8.25 & 4.06 & High Myopia \\
6 & 55 & M & 0.7 & 1.5 & -13.125 & 3.36 & High Myopia \\
7 & 62 & F & 1.2 & 0.75 & -3.575 & 4.19 & BRVO Acute Glaucoma \\
8 & 72 & F & 0.7 & 0.75 & -2.575 & 3.53 & Keratoconus \\
9 & 23 & M & 0.1 & 1.5 & -6.50 & 4.22 & NTG \\
10 & 45 & F & 0.6 & 1 & -13.875 & 4.06 & NTG, High Myoia \\
11 & 45 & F & 0.5 & 1.25 & -14.75 & 4.29 & High Myopia \\
12 & 45 & M & 0.02 & 1.5 & -14.5 & 4.62 & Fundus Albipunctatus \\
13 & 61 & F & 0.8 & 2.25 & -8.75 & 4.58 & Fundus Albipunctatus \\
14 & 61 & F & 0.9 & 2 & -7.75 & 3.31 & NTG \\
15 & 63 & F & 0.9 & 0.75 & -2.1 & .58 \\
\hline
\end{tabular}

Abbreviations: BRVO, branch retinal vein occulusion; CDVA, corrected distance visual acuity; CC, cornal cylinder; High Myopia, high myopia with axial length greater than 28mm; NTG, nomal tension glaucoma; SE, spherical equivalent. 


\section{Postoperative outcome}

The mean postoperative manifest spherical equivalent was $-0.11 \pm 0.54 \mathrm{D}(-0.75$ to $0.625 \mathrm{D})$, the mean corneal cylinder was $1.54 \pm 0.53 \mathrm{D}(0.75$ to $2.5 \mathrm{D})$, and the manifest cylinder was $0.36 \pm 0.66 \mathrm{D}(0$ to $1.75 \mathrm{D})$; the toric model was implanted in 13 of the 15 eyes.

The UDVA, CDVA, UNVA, and CNVA at 1 day and 6 months postoperatively are shown in Figure 1. The mean UDVA and CDVA were $-0.016 \pm 0.12$ and $-0.08 \pm 0.09 \log \mathrm{MAR}$, respectively, at 1 day postoperatively, and all eyes registered better than 0.18 both in UDVA and CDVA. Of the 15 operated eyes, 53\% registered 0 or better in UDVA, $87 \%$ registered 0 or better in CDVA (Figure 1a), and the mean UDVA and CDVA were $-0.001 \pm 0.11$ and $-0.07 \pm 0.11$, respectively, at 6 months postoperatively (Figure 1b). The mean UNVA and CNVA were $0.19 \pm 0.17$ and $-0.03 \pm 0.09$ $\log \mathrm{MAR}$, respectively, at 1 day postoperatively (Figure 1c), and $0.2 \pm 0.19$ and $-0.01 \pm 0.12 \log M A R$, respectively, at 6 months postoperatively; $73 \%$ of the eyes registered better than 0 in CNVA. Only two eyes achieved better than 0 in UNVA, and $20 \%$ of the eyes registered worse than 0.3 in UNVA (Figure 1d).
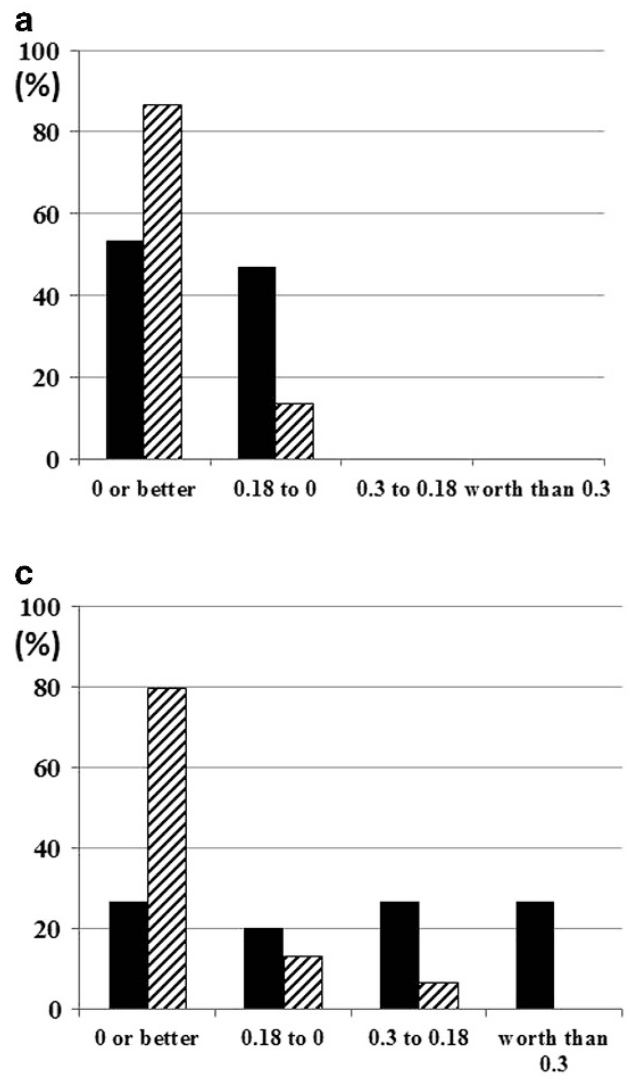

The mean monocular defocus curves are shown in Figure 2a. As shown in the figure, there were two peaks of maximum vision located at far and near focus, with reduced VA for intermediate distances. The far peak of the monocular curves was $-0.07 \pm 0.13(\log$ MAR), and the peak of optimum near vision was $0.15 \pm 0.19(\log M A R)$ at the variance of $-2.5 \mathrm{D}$ which was equivalent of $40 \mathrm{~cm}$ from the eye.

Values of near to intermediate $\mathrm{VA}$ are shown in Figure $2 \mathrm{~b}$. Both uncorrected and distance-corrected VA registered the best acuity at $50 \mathrm{~cm}$ but registered 0.21 $\log$ MAR or better at all distances. Moreover, the mean uncorrected VA registered $0.14 \log \mathrm{MAR}$ at $30 \mathrm{~cm}, 0.12$ $\log$ MAR at $40 \mathrm{~cm}$, and $0.10 \log$ MAR at $70 \mathrm{~cm}$. Both uncorrected and distance-corrected VA did not show a marked decrease in intermediate VA.

Contrast sensitivity registered higher at three and six cycles per degree and lower at 18 cycles per degree than the lower boundary of normal healthy subjects aged 2060 years, as provided by VectorVision, the manufacturer of the contrast sensitivity test scale (Figure 3), and was comparable to previously reported data of MPlusimplanted healthy eyes. ${ }^{7-10}$

b

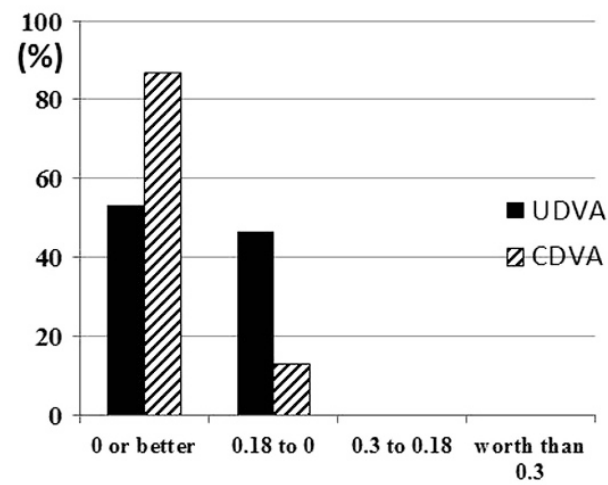

d

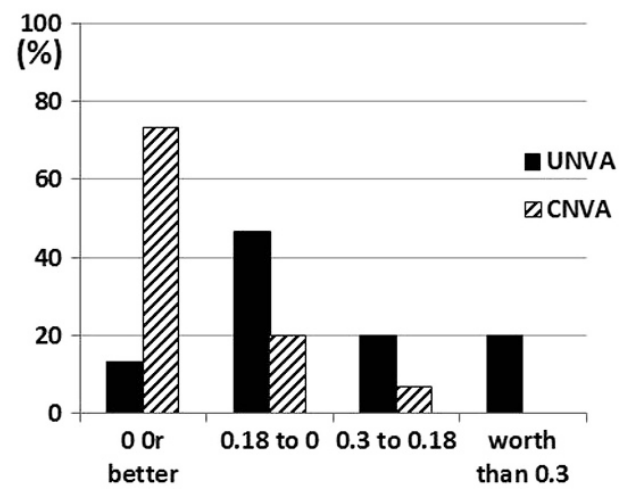

Figure 1 Corrected and uncorrected distance visual acuity (VA) at 1 day (a) and 6 months (b) postoperative. Corrected and uncorrected near VA at 1 day (c) and 6 months (d) postoperative (UDVA, uncorrected distance VA; CDVA, corrected distance VA; CNVA, corrected near VA; UNVA, uncorrected near VA). 
a

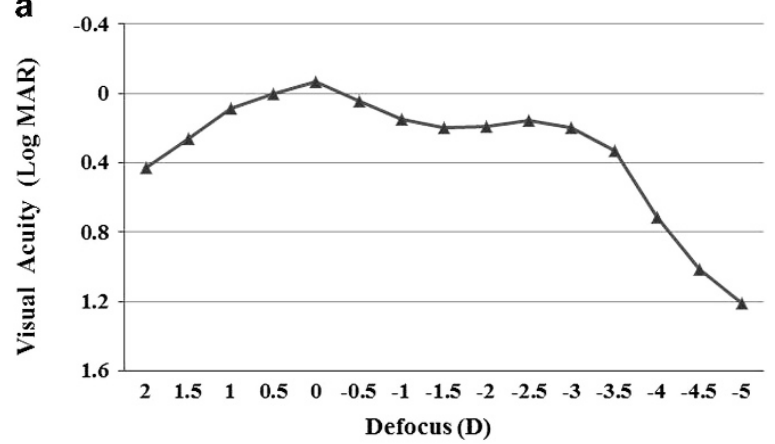

b

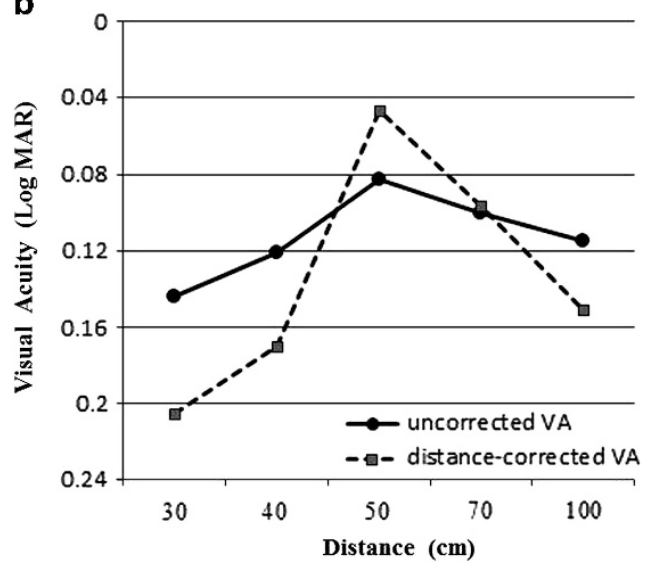

Figure 2 Mean monocular defocus curve after Lentis MPlus IOL implantation (a) Peaks of maximum VA were seen approximately at the 0.00 and $-2.50 \mathrm{D}$. Defocus curve indicates same feature as this intraocular lens, even implanted into complicated eyes. Mean monocular uncorrected and distance-corrected VA at near to intermediate distances (b) This direct measurement of near to intermediate distance VA also revealed the feature of this refractive MIOL as well as defocus curve. Both uncorrected and distance-corrected VA registered the best acuity at $50 \mathrm{~cm}$ but registered $0.21 \log$ MAR or better at all distances $(\mathrm{IOL}=$ intraocular lens, VA = visual acuity)

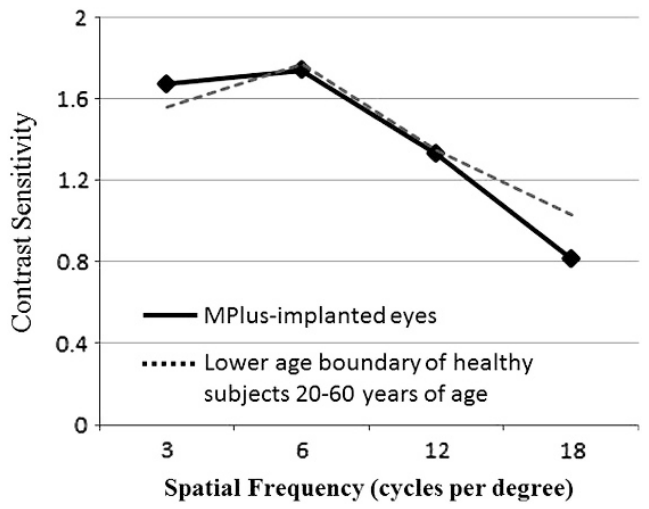

Figure 3 Mesopic log contrast sensitivity function at far distance. Contrast sensitivity registered higher at three and six cycles per degree and lower at 18 cycles per degree than the lower age boundary of normal healthy subjects from 20 to 60 years of age.
Of the 11 patients, all were completely independent of spectacles for distance vision. For near vision, one patient needed to use eyeglasses occasionally, and one patient needed to use eyeglasses constantly. For distance vision, 11 patients $(100 \%)$ rated their quality of vision as 4 or higher (very good or good) among five items, and seven patients (64\%) rated it at 5 (very good). For near vision, 11 patients $(100 \%)$ rated their quality of vision as 3 (acceptable) or higher and three $(27 \%)$ rated it at 5 (very good). None of the patients rated their quality of vision as 1 or 2 (very poor or poor) for both distance and near vision (Figure 4). Two patients (20\%) reported having 'hazy vision'; however, neither of those two patients reported that the haziness caused them any difficulty.

\section{Discussion}

LENTIS MPlus sectorial addition refractive MIOLs were implanted into 15 eyes with cataract complicated with a coexisting ocular pathology, and the clinical results were evaluated. All operated eyes achieved good UDVA with useful near vision from the early postoperative period. All distance VA tests revealed good uncorrected VA at any distance from near to intermediate $(30 \mathrm{~cm}$ to $1 \mathrm{~m})$. The findings of this study revealed that the refractive MIOL profile was maintained, even in cataract eyes complicated with coexisting ocular diseases.

The registered comparative contrast sensitivity findings in this case series are similar to those previously reported of diffractive MIOLs implanted into cataract eyes with no coexisting ocular pathology. ${ }^{4}$ The LENTIS MPlus MIOL is designed to overcome the drawbacks of other MIOLs by providing high contrast sensitivity and minimizing halo and glare, ${ }^{12}$ a design that is based on the concept of refractive rotational asymmetry. Reportedly, the aim of the technology is to reduce sources of light scattering and aberrations. ${ }^{8}$ In fact, both Muñoz et $a l^{7}$ and Alió et al ${ }^{8-10}$ reported that contrast sensitivity in MPlus-implanted patients was similar to that in monofocal IOL-implanted patients at any spatial frequency. ${ }^{7-10}$ Moreover, Alió et al ${ }^{9,10}$ reported that eyes implanted with an MPlus MIOL registered higher contrast sensitivity than did eyes implanted with a diffractive MIOL. This feature might also contribute especially for good distance vision, ${ }^{7}$ and the immediate visual rehabilitation shown in our results at 1 day postoperatively.

The results of our patient questionnaire revealed an excellent rate of patient satisfaction with regard to postoperative distance vision, which agrees with the results of the above-referenced previous reports. In addition, the rate of spectacle independence was very similar to that of a previous report of MPlus-implanted healthy eyes, ${ }^{7}$ thus indicating that the multifocal 
Use of Spectacles (Far)

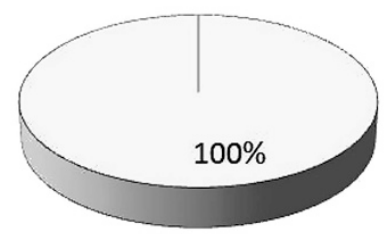

Satisfaction for Far Vision

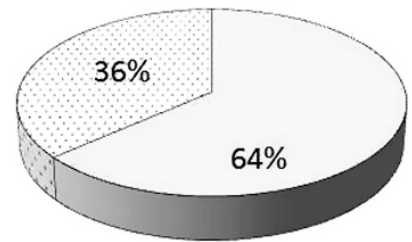

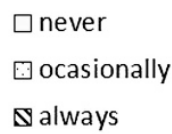

\$always

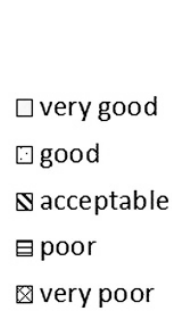

Use of Spectacles (Near)

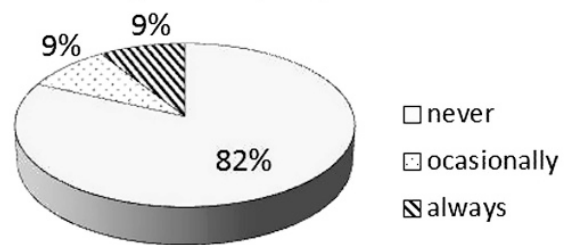

Satisfaction for Near Vision

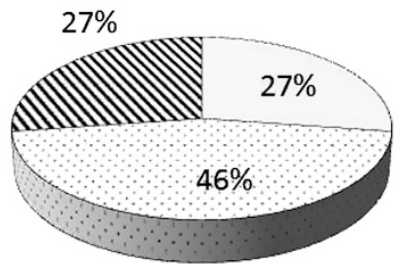

Figure 4 Postoperative spectacle independency and patient satisfaction rate from questionnaire.

mechanism of this MIOL does not decrease the quality of vision, even in complicated eyes.

Refractive MIOLs were introduced in 1995 in Japan, followed by diffractive MIOLs such as the Alcon ReSTOR IOL (Alcon, Fort Worth, TX, USA) or the Abbott Medical Optics TECNIS Multifocal IOL (Abbott Laboratories Inc. Abbott Park, IL, USA), and a large proportion of MIOLs currently used are diffractive MIOLs for the above-mentioned reasons. However, surgeons occasionally come across cases that complain of hazy vision caused by a decrease of contrast accompanied with diffraction phenomena, ${ }^{4-6,14}$ even though the eye in which the MIOL is implanted is otherwise healthy except for cataract. ${ }^{4}$ With that aside, it is still difficult to predict preoperatively which cases might experience a postoperative outcome of hazy vision. Moreover, it is often stated that complicated cataract eyes are a contraindication of diffractive MIOL implantation. However, the exact branching point of indication and contraindication remains unclear. On the other hand, the relatively high contrast of refractive MIOLs is well reported, 9,10 and this feature should be considered in the future for indication or selection of MIOL implantation. de Wit et al ${ }^{13}$ implanted the LENTIS MPlus MIOL bilaterally in the eyes of 14 anisometropic amblyopic patients. The results of their study showed improvements in VA, binocular function, reading speed, and quality of life score, with no significant side effects. Eyes having diseases other than cataract itself present a handicap for postoperative VA; thus, preservation of contrast sensitivity is an important goal in MIOL implantation in such cases.

Comparative controlled clinical trials are virtually impossible in such case series, for, as shown in this study, the coexisting ocular pathologies included only one corneal disease, glaucoma, and three kinds of retinal disorder. More objective evidence with a larger number of cases and pertaining to other ocular complications, as well as long-term evaluation, is required. In addition, careful consideration is needed, especially in cases with a potential progressive pathology. ${ }^{15}$

In addition, a more clear designation of the indication for refractive MIOL implantation needs to be established for each disease. Moreover, there is an urgent need to clarify the indication for MIOL implantation in such cases owing to the increasing needs for presbyopia correction.

In conclusion, use of the LENTIS MPlus sectorial refractive MIOL for cataract eyes complicated with ocular pathologies can achieve good UNVA with no significant adverse effect. It is possible that, when performed with careful case selection, implantation of refractive MIOLs is relatively suitable for patients who have eye diseases other than cataract yet wish to have a life independent of the need to wear glasses. Thus, these findings might contribute to an expansion of the number of patients who can benefit from this premium MIOL technology.

\section{Summary}

What was known before

- Refractive multifocal IOLs (MIOLs) can achieve uncorrected distance and near visual acuity with less dropoff of intermediate vision, and can contribute to independence from spectacles.

- Sectorial refractive MIOLs have less light scattering and provide high contrast sensitivity.

What this study adds

- Implantation of sectorial refractive MIOLs in cataract eyes complicated with coexisting ocular pathologies provides excellent distance vision and good near vision with comparative glass-wear independence and no side effects. 


\section{Conflict of interest}

The authors declare no conflict of interest.

\section{Acknowledgements}

We thank John Bush for reviewing the manuscript.

\section{References}

1 Chang DF. Prospective functional and clinical comparison of bilateral ReZoom and ReSTOR intraocular lenses in patients 70 years or younger. J Cataract Refract Surg 2008; 3: 934-941.

2 Pieh S, Lackner B, Hanselmayer G, Zöhrer R, Sticker M, Weghaupt $\mathrm{H}$ et al. Halo size under distance and near conditions in refractive multifocal intraocular lenses. Br J Ophthalmol 2001; 85: 816-821.

3 Artigas JM, Menezo JL, Peris C, Felipe A, Díaz-Llopis M. Image quality with multifocal intraocular lenses and the effect of pupil size: comparison of refractive and hybrid refractivediffractive designs. J Cataract Refract Surg 2007; 33: 2111-2117.

4 Blaylock JF, Si Z, Aitchison S, Prescott C. Visual function and change in quality of life after bilateral refractive lens exchange with the ReSTOR multifocal intraocular lens. J Refract Surg 2008; 24: 265-273.

5 Vingolo EM, Grenga P, Iacobelli L, Grenga R. Visual acuity and contrast sensitivity: AcrySof ReSTOR apodized diffractive versus AcrySof SA60AT monofocal intraocular lenses. J Cataract Refract Surg 2007; 33: 1244-1247.

6 Souza CE, Muccioli C, Soriano ES, Chalita MR, Oliveira F, Freitas LL et al. Visual performance of AcrySof ReSTOR apodized diffractive IOL: a prospective comparative trial. Am J Ophthalmol 2006; 141: 827-832.

7 Muñoz G, Albarrán-Diego C, Ferrer-Blasco T, Sakla HF, García-Lázaro S. Visual function after bilateral implantation of a new zonal refractive aspheric multifocal intraocular lens. J Cataract Refract Surg 2011; 37: 2043-2052.
8 Alió JL, Piñero DP, Plaza-Puche AB, Chan MJ. Visual outcomes and optical performance of a monofocal intraocular lens and a new-generation multifocal intraocular lens. J Cataract Refract Surg 2011; 37241-37250.

9 Alió JL, Plaza-Puche AB, Javaloy J, Ayala MJ, Moreno LJ, Piñero DP. Comparison of a new refractive multifocal intraocular lens with an inferior segmental near add and a diffractive multifocal intraocular lens. Ophthalmology 2012; 119: 555-563.

10 Alió JL, Plaza-Puche AB, Javaloy J, Ayala MJ. Comparison of the visual and intraocular optical performance of a refractive multifocal IOL with rotational asymmetry and an apodized diffractive multifocal IOL. J Refract Surg 2012; 28: 100-105.

11 Pepose JS, Quzi MA, Davies J, Doane JF, Loden JC, Sivalingham $\mathrm{V}$ et al. Visual performance of patients with bilateral vs combination Crystalens, ReZoom, and ReSTOR intraocular lens implantation. Am J Opthalmol 2007; 144: 347-357.

12 Rocha KM, Chalita MR, Souza CE, Soriano ES, Freitas LL, Muccioli $\mathrm{C}$ et al. Postoperative wave-front analysis and contrast sensitivity of a multifocal apodized diffractive IO (ReSTOR) and three monofocal IOLs. J Refract Surg 2005; 21: 808-812.

13 de Wit DW, Diaz JM, Moore TC, Moore JE. Refractive lens exchange for a multifocal intraocular lens with a surfaceembedded near section in mild to moderate anisometropic amblyopic patients. J Cataract Refract Surg 2012; 38: 1796-1801.

14 Souza CE, Gerente VM, Chalita MR, Soriano ES, Freitas LL, Belfort R Jr. Visual acuity, contrast sensitivity, reading speed, and wavefront analysis: pseudophakic eye with multifocal IOL (ReSTOR) versus fellow phakic eye in non-presbyopic patients. J Refract Surg 2006; 22: 303-305.

15 Braga-Mele Rosa, Chang David, Dewey Steven, Foster Gary, An Henderson Bonnie, Hill Warren et al. Multifocal intraocular lenses: Relative indications and contraindications for implantation. J Cataract Refract Surg 2014; 40: 313-322. 